\title{
Eribulin: clinical review and new perspective in advanced breast cancer
}

\author{
Arvind Gadekar* \\ Medical Services, Emcure Pharmaceuticals Limited, Rajiv Gandhi IT Park, Hinjawadi, Pune, Maharashtra, India
}

Received: 01 March 2021

Revised: 06 April 2021

Accepted: 07 April 2021

\section{*Correspondence:}

Dr. Arvind Gadekar,

E-mail: arvind.vilas@emcure.co.in

Copyright: $\odot$ the author(s), publisher and licensee Medip Academy. This is an open-access article distributed under the terms of the Creative Commons Attribution Non-Commercial License, which permits unrestricted non-commercial use, distribution, and reproduction in any medium, provided the original work is properly cited.

\begin{abstract}
Eribulin is a unique microtubule inhibitor with mitotic and nonmitotic mechanisms of action. Eribulin has substantial activity in patients with pretreated (anthracycline and a taxane) advanced or metastatic breast cancer was confirmed by large-scale phase III clinical trials. We review recent pharmacological and clinical findings of eribulin use in metastatic breast cancer, particularly highlighting eribulin in difficult-to-treat and aggressive disease and safety data in specific patient populations. Furthermore, recent advancements and potential future directions for its clinical usage in our understanding are discussed. Ongoing studies of eribulin in combination with immunotherapies and established cytotoxic agents may facilitate to shape the future landscape in the treatment of breast cancer.
\end{abstract}

Keywords: Eribulin, Mechanism of action, Metastatic breast cancer, Microtubule inhibitor, Triple-negative breast cancer

\section{INTRODUCTION}

Breast cancer $(\mathrm{BC})$ is the second most frequently diagnosed cancer globally, with a frequency adding up to $11.9 \%$. It is also the first most frequently occurring cancer among women comprising $25.2 \%$ of all newly-diagnosed cancers. ${ }^{1-3}$ It is assumed that one in eight women in the world will develop mammary gland cancer and that only $5-10 \%$ of all cases of this cancer are caused by genetic disorders, whereas the remaining $90-95 \%$ of cases are connected to environmental factors and lifestyle. ${ }^{4,5}$ Despite treatment advances for metastatic disease (chemotherapeutic agents, hormonal therapies and targeted agents) that have led to improved outcomes for patients with metastatic breast cancer (MBC), the overall prognosis remains poor, with the 5 year survival rate approximating only $25 \% .^{6}$ Treatment strategies for patients with metastatic disease should consider many parameters, such as molecular subtype (luminal or basal, HER2-enriched BC), the event-free interval, prior treatments, the extent and the site of disease, patient tolerability and preference. As TNBC lacks a specific molecular target, the chemotherapy is the mainstay of therapy. Taxane and anthracycline-based regimens are the standard of care in the management of BC and despite the high response rate, treatment failure can occur in the majority of patients. The need for alternative compounds with antineoplastic activity and a safe toxicity profile is paramount. Eribulin is a novel compound approved for patients with locally advanced or metastatic cancer who have progressed after at least one chemotherapeutic regimens for the treatment of advanced $\mathrm{BC}$ after progression on anthracycline and taxanes. In this narrative review, we will discuss the role of the drug eribulin in the treatment of MBC. ${ }^{7}$

\section{UNDERSTANDING ERIBULIN'S MECHANISM OF ACTION}

Eribulin exhibits its action by inhibiting the dynamics of the microtubules. It binds to the plus ends of the microtubules and suppresses the microtubule growth in the interphase without showing the effect on the shortening phase and isolates tubulin into nonproductive aggregates, leading to $\mathrm{G} 2 / \mathrm{M}$ cell-cycle block and ultimately apoptosis after prolonged mitotic blockage. ${ }^{8}$ Its mechanism is unique 
from other microtubule inhibitors like vinca alkaloids and taxanes, which affect both the shortening and growing phases. Distinctly, while vinca alkaloids bind at both the ends (alfa and beta), taxanes and epothilones bind at the beta end and specifically on the inner aspect of the microtubule. Another unique feature is that eribulin binds either at the interface between the alfa and beta subunits of the microtubule or the beta subunit alone and is therefore now being studied extensively in the treatment of patients with taxane-resistant breast cancer arising due to betatubulin mutations. ${ }^{9}$ In addition to its antimitotic mechanism, in preclinical cancer models, eribulin has shown nonmitotic effects including vascular remodeling, the reversal of the epithelial-to-mesenchymal (EMT) transition and suppression of cancer cell migration, invasion and experimental metastasis. ${ }^{10-12}$ In preclinical xenograft models of human breast cancer, eribulin produced tumor vascular remodeling leading to increased perfusion of tumor cores. ${ }^{11}$ This was demonstrated by an increase in the tumor perfusion transfer coefficient visually, using dynamic contrast-enhanced MRI, increased Hoechst dye perfusion and decreases in staining for carbonic anhydrase, an established marker of hypoxia. ${ }^{9}$ These effects were linked with increased numbers of micro vessels in tumors that had been exposed to a single dose of eribulin approximately 1 week earlier. Furthermore, prior treatment with eribulin enhanced the antitumor activity of capecitabine in the MDA-MB-231 xenograft model. These findings suggest that eribulin-induced vascular remodeling leads to changes in the tumor microenvironment that reduce or eliminate hypoxia-driven growth aggressiveness and increase the exposure of subsequent treatments. ${ }^{13}$

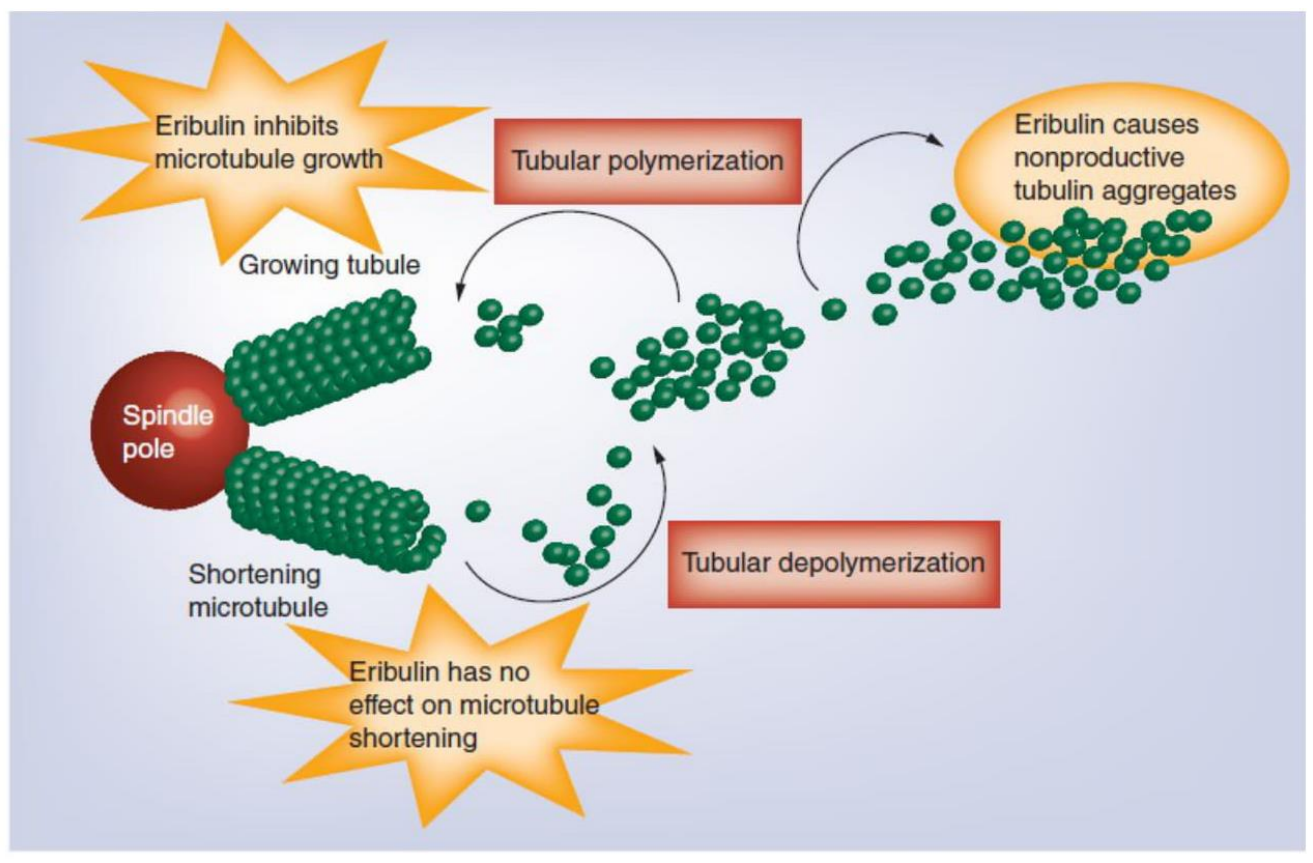

Figure 1: Eribulin mechanism of action. ${ }^{7}$

\section{PRECLINICAL ACTIVITY AND PHARMACO- KINETICS}

Early preclinical studies have shown eribulin to inhibit invitro growth of different kinds of human cancer cell lines. In BC cell line MDA-MB-435 the cell growth inhibition was achieved at lower concentrations than either paclitaxel or vinblastine. ${ }^{14}$ In tumor xenograft models eribulin exhibited tumor regression and remission with life span gain at dosing less than maximum tolerated dose (MTD) with a wide therapeutic window and greater potency compared other anticancer drugs such as paclitaxel. ${ }^{4}$ Eribulin demonstrated a triexponential elimination from the plasma of patients receiving a rapid intravenous infusion (over 1-2 min). After a rapid distributive phase, it is slowly eliminated with a terminal elimination half-life in the range of $36-48$ hours. ${ }^{15}$ Eribulin exhibits linear pharmacokinetics with a mean elimination half-life of $\sim 40$ hours. Accumulation of eribulin was not observed with weekly administration. ${ }^{16}$ Additionally, the drug eribulin was predominantly metabolized by CYP3A4. However, eribulin was not shown to affect the metabolism of other therapeutic agents which are metabolized by CYP3A. ${ }^{17}$ The drug is mainly eliminated in feces and $<10 \%$ in urine, mostly as unchanged. ${ }^{16}$ Hepatic impairment resulted in reduced clearance and prolonged elimination half-life of the drug. ${ }^{18}$ Renal impairment reduced eribulin clearance as well, thus enhancing exposure. Pharmacokinetic data provide drug's dose reduction in patients with moderate or severe renal impairment. ${ }^{19}$ On estrogen-stimulated receptor-positive $\mathrm{BC}$ cells eribulin demonstrated strong antitumor effects while the association of the drug with an antiestrogen caused only a weakly additive antitumor effect. Eribulin exhibited anticancer stem cell effects on estrogen receptor, positive as well as negative BC cells. ${ }^{20}$ Additionally, a dose-related inhibition of Akt activation and cell growth inhibition was observed in triple-negative 
BC (TNBC) and HER2 cell lines when administered alone or combined with RAD001. ${ }^{21}$

\section{REVIEW OF CLINICAL TRIALS}

\section{Phase I trial}

The maximum tolerated dose, dose-limiting toxicities and pharmacokinetics of eribulin were analyzed in patients with advanced solid tumors. In conclusion, a dose of 2 $\mathrm{mg} / \mathrm{m}^{2}$ eribulin administered over a period of 1 hour had a manageable toxicity profile and dose escalation was related to increased neutropenia. ${ }^{22}$

\section{Phase II trial}

Based on the mechanism of action of eribulin on microtubules and its activity in breast cancer cell lines, it was hypothesized that this drug may have an action in refractory BC. ${ }^{23}$ In a Japanese phase 2 study, eribulin was utilized in heavily pretreated MBC patients. The overall response rate (ORR) in this study was $21.3 \%$, with progression-free survival (PFS) of 3.7 months and overall survival (OS) of 11.1 months. ${ }^{24}$ In a single-arm, openlabelled, phase 2 study by Cortes et al eribulin was used in locally advanced and metastatic breast cancer patients previously treated with an anthracycline, taxane, and capecitabine. ${ }^{25}$ ORR reported in this study was $14.1 \%$, with a median duration of response of 4.1 months, PFS of 2.6 months and OS of 10.4 months. Neutropenia was observed in 54\%, fatigue in $10 \%$ and peripheral neuropathy in $6.9 \%$ of patients. In a phase 2 study by Linda et al eribulin was studied in breast cancer patients who were previously treated with an anthracycline and taxane and served an OS of 9 months with a comparable toxicity pattern of neutropenia and neuropathy. ${ }^{26}$

\section{Phase III clinical trial}

In the EMBRACE study, the first phase III global, multicenter, randomized, open-label study, 763 heavily pretreated $\mathrm{MBC}$ patients were randomly assigned with a $2: 1$ ratio to receive either eribulin mesylate $1.4 \mathrm{mg} / \mathrm{m}^{2}$ on days 1 and 8 every 21 days or treatment of physician's choice (TPC). The definition of TPC was any single-agent chemotherapy, hormonal or biological treatment, radiotherapy or supportive care. No patients in the TPC group received supportive care. The primary objective of the study was to compare OS in both the treatment arms, secondary end-points were PFS, ORR and DoR. The median age of the enrolled patients was 55 years, $16 \%$ had HER2-positive disease, more than half (51\%) were observed to be having three or more organs involved, the median number of previous chemotherapy lines was four (range 1-7), almost all had received an anthracycline and a taxane and almost $70 \%$ had received capecitabine. In total 508 patients who received eribulin and 254 received with TPC there was significant improvement observed in OS in women treated with eribulin (median 13.1 months, 95\% CI was 11.8-14.3) compared with those in the TPC group
(10.6 months, 9.3-12.5; hazard ratio 0.81 , 95\% CI was $0.66-0.99 ; p=0.041)$. This difference was maintained at the updated analysis (not protocol pre-specified), based on a $77 \%$ event rate, requested by European and US regulatory authorities. Notwithstanding this improvement in OS, median PFS was not significantly longer with eribulin than with TPC (3.7 versus 2.2 months, respectively, $\mathrm{p}=0.137$ ). Significant improvement was noted in the objective response rate by the treatment with eribulin compared with TPC (12 versus $5 \%$, respectively, $\mathrm{p}=0.002$ ). The majority of AEs fell in grade 1/2. Patients in the eribulin arm were observed to be having more grade $3 / 4$ AEs than the TPC patients arm; neutropenia, leucopenia and peripheral neuropathy were the most commonly observed AEs. Peripheral neuropathy (any grade) was the most common AE leading to discontinuation of eribulin, in 24 (5\%) of 503 patients. ${ }^{27}$ This was a pivotal study demonstrating a survival advantage in heavily pretreated MBC patients by the usage of eribulin. The choice of the comparator, at physician discretion, was an important factor because it shows the real-world scenario and represents the strength of the trial. The improved OS without PFS prolongation is an issue too. This hypothesis cannot be either confirmed or excluded, it must be emphasized that the main goal of the most active drug would be a delay in cancer progression as well as prolongation of survival. Another possible explanation could be based on eribulin-induced vascular remodeling. Increased tumor perfusion might have led to the better activity of subsequent drugs by both reducing hypoxia-driven chemo resistance and enhancing intratumoral delivery of the drugs. Moreover, Funahashi et al in advanced BC drug evaluation animal models, eribulin showed that eribulin-pretreated tumors were more sensitive to subsequent capecitabine treatment than nonpretreated tumors. ${ }^{11}$ Pooled data from two single-arm phase II studies and one phase III randomized trial demonstrated that the treatment with eribulin may be useful for selected older patients. ${ }^{28}$ Another phase III trial was recently published. ${ }^{29}$ MBC patients, who have received up to three prior chemotherapy regimens for advanced disease, including anthracyclines and taxanes, were eligible. Overall, 1102 patients were enrolled to receive either eribulin mesylate $1.4 \mathrm{mg} / \mathrm{m}^{2}$ on day 1 and 8 or capecitabine $1250 \mathrm{mg} / \mathrm{m}^{2}$ twice per day on days $1-14$, both in 21-day cycles. PFS and OS were co-primary endpoints of this study. Secondary end-points included ORR, DoR, 1, 2 and 3 year survival, safety, quality of life and population pharmacokinetic/pharmacodynamics relationship. Overall the majority of patients (72\%) received the study drug as their first-line or second-line treatment for metastatic disease. Almost 90\% had visceral disease with involvement of two or more sites in more than $80 \%$ of patients. The study failed to show either of its coprimary endpoints. A trend in OS for patients treated with eribulin (15.9 versus 14.5 months) was seen, but this difference only approached statistical significance $(p=0.056)$. PFS was the same in both study arms (4.1 versus 4.2 months for eribulin and capecitabine, respectively). ORRs by the independent review were the same, 11 and $11.5 \%$ for eribulin and capecitabine 
respectively. No unexpected safety concern was noted. No difference was observed from the analysis of quality of life. A recent published pooled analysis of 305/EMBRACE and study 30130 analyzed OS, PFS and response rates in the intent-to-treat population and selected subgroup. Overall 1062 patients received eribulin and 802 control. The observed median OS was 15.2 months with eribulin and 12.8 months with control (hazard ratio HR was $0.85 ; 95 \%$ CI was $0.77,0.95 ; \mathrm{p}=0.003$ ). Treatment with eribulin significantly favored OS and this finding was noted across all patient subgroups. In particular, in women with HER2-negative disease, a 2.9 month difference in OS was observed (median OS 15.2 versus 12.3 months) with eribulin and control, respectively; HR was $0.82 ; \mathrm{p}=0.002$ ), there was no statistical significance in HER2-negative, HR-positive disease $(p=0.060)$. In the HER2-positive population, there was a trend in OS favoring eribulin treatment. TNBC gained major improvements in survival. Median survival was 4.7 months longer in patients treated with eribulin than in those treated with control (median OS 12.9 versus 8.9 months, $\mathrm{HR}$ was $0.74 ; \mathrm{p}=0.006$ ). Interestingly patients with more than two organs involved and patients not refractory to taxanes had improved survival (median OS: 13.1 versus 10.5 months; HR was 0.77; $\mathrm{p}<0.001$ and 17.4 versus 14.4 months; HR was 0.81 ; $\mathrm{p}=0.007$ ). Pivot et al in a very recent pooled analysis of the 301 and 305 studies aimed to evaluate the 2014 EU indication for eribulin use in earlier lines, confirmed previous data. ${ }^{31}$ The data emphasized the benefit of eribulin over the control arm in terms of OS. Significantly longer median OS in patients treated with eribulin was observed, being 15.0 months and 12.6 months in eribulin and in the control arm, respectively (HR was $0.85 ; 95 \% \mathrm{CI}$ was $0.76-0.94 ; \mathrm{p}<0.01)$. PFS was considerably longer being 3.9 months and 3.2 months (HR was $0.87 ; 95 \% \mathrm{CI}$ was $0.78-0.97 ; \mathrm{p}<0.05)$ in eribulin and the control arm, respectively. The benefit in OS was seen in HER2negative, TNBC, ER-negative, those without TNBC, with more than two sites involved and in patients with visceral involvement, without increased toxicity. ${ }^{31}$

\section{REAL-LIFE DATA}

Observational real-world studies have demonstrated efficacy and tolerability results for eribulin treatment similar to those reported in clinical trials. Clinical benefit has been reported in a large 574 unselected patients with MBC, heavily pretreated patients with MBC, elderly patients $\geq 70$ years, heavily pretreated elderly patients with locally recurrent/MBC, metastatic TNBC, ER-positive $\mathrm{MBC}$, hormone-refractory breast cancer, taxane-refractory $\mathrm{MBC}$ and in patients with brain metastases. ${ }^{28,32-41}$ In a retrospective, real-world analysis of 252 patients with metastatic TNBC, it was observed that the estimated realworld OS of 14.7 months for late-line eribulin users was similar to the 13.1 months reported in EMBRACE. ${ }^{36,42}$ In a retrospective analysis of observations from study 301, six patients with brain metastases (three each treated with eribulin or capecitabine) were studied as a case series. ${ }^{41}$ All three patients treated with eribulin received six cycles and demonstrated some degree of decrease in the size of their brain lesions during treatment. The three patients on capecitabine had different outcomes related to brain metastases; one patient had an increase in the size of brain lesions, one patient had no observable change in the size of brain lesions and one patient had a complete response that was not confirmed with a follow-up scan. In a recent case study report of two patients with breast cancer and brain metastases eribulin treatment, either following whole-brain radiotherapy (WBRT) or surgical resection of the tumor, resulting in clinical regression. ${ }^{43}$ One patient (with HER2-negative breast cancer) was given WBRT followed by eribulin monotherapy. After eight cycles of treatment, the metastatic brain tumors demonstrated regression with systematically stable disease. The second patient (hormone receptor-negative, HER2-positive breast cancer) was given eribulin with concurrent WBRT after surgical resection of the tumor. After 23 cycles of eribulin, the patient demonstrated no tumor recurrence or progression in the brain. ${ }^{43}$ Although suggestive of a clinical benefit to patients with $\mathrm{BC}$ who develop brain metastases, future studies are required to evaluate the effects of eribulin in this subset of patients.

\section{SAFETY PROFILE}

In the landmark EMBRACE study, which included patients with heavily pretreated MBC, neutropenia was observed in $52 \%$ of patients in the eribulin arm and was managed with dose delays, reductions and the administration of growth factors (granulocyte colonystimulating factor in $18 \%$ of patients). ${ }^{27}$ The incidence of severe neutropenia (absolute neutrophil count $<500 / \mathrm{mm}^{3}$ ) lasting more than 1 week occurred in 12\% (62/503) of patients and caused treatment discontinuation in less than $1 \%$ of patients. Febrile neutropenia was seen in $5 \%$ $(23 / 503)$ of patients, with two patients $(0.4 \%)$ dying from complications of febrile neutropenia. ${ }^{13}$ Grade $3 / 4$ neutropenia has also been observed in real-world studies of eribulin in patients with heavily pretreated MBC with rates ranging from $22 \%$ (17/78) to $49 \%$ (404/827). ${ }^{33}$ An unselected population of patients receiving eribulin in a real-world setting were reported with grade 3/4 neutropenia in $12.2 \%(70 / 574) .{ }^{32}$ In EMBRACE, peripheral neuropathy was the most common toxicity causing treatment discontinuation $(5 \%$ of patients, $24 / 503)$, with grade 3 peripheral neuropathy occurring in $8 \%(40 / 503)$ of patients and grade 4 in $0.4 \%(2 / 503)$ of patients. ${ }^{4}$ Grade $3 / 4$ peripheral neuropathy has also been observed at rates of $5 \%(4 / 78)$ to $7 \%(57 / 827)$ of patients among those with heavily pretreated MBC. ${ }^{33,28}$ In an unselected population, the rate of grade 3/4 neuropathy was $2.6 \%(15 / 574) .{ }^{33}$ Alternative schedules of eribulin administration are being analyzed to enable patients who are sensitive to myelosuppression to tolerate the treatment better. In comparison to the licensed dosing schedule of eribulin administered on days 1 and 8 every 3 weeks, a modified biweekly regimen gives additional time for hematologic recovery between treatment administrations, which may improve eribulin's safety profile. ${ }^{44}$ In a 
prospective phase II trial in Japan in patients with pretreated MBC, a biweekly plan of eribulin $\left(1.4 \mathrm{mg} / \mathrm{m}^{2}\right.$ on days 1 and 15 of a 28 day cycle) was tolerable and had comparable efficacy in patients who were not tolerant to the standard eribulin schedule. In that study, it was observed that the patients on the biweekly schedule had a mean relative dose intensity of $62.7 \%$, ORR of $21 \%$ and a median OS of 523 days. ${ }^{45}$ A phase II trial in patients with MBC evaluating the incidence of peripheral neuropathy with standard-regimen eribulin versus ixabepilone showed that, although not statistically significantly different, fewer patients receiving eribulin discontinued treatment due to neuropathy when compared to those who received ixabepilone (3.9 versuus $18.0 \%){ }^{46}$

The time to onset of neuropathy was 35.9 weeks for eribulin compared with 11.6 weeks for ixabepilone. Taken together, these observations show that neuropathy associated with eribulin tended to occur later and patients treated with eribulin remained on treatment for a longer duration. The presence of liver metastasis is usually a challenge for patients of breast cancer receiving treatment in later-line settings. Pharmacokinetic studies have demonstrated that eribulin is predominantly excreted unchanged (not metabolized), with only 5-6\% of the administered dose eliminated through the urine. ${ }^{21,47}$ In patients with solid tumors and mild-to-moderate liver impairment, eribulin dose reductions are recommended. ${ }^{13,18}$ Liver impairment enhanced exposure to eribulin in patients with child-Pugh class A impairment (1.75-fold higher) and child-Pugh class B impairment (2.48-fold higher). ${ }^{18}$ More analyses are needed further to determine the safety and efficacy of eribulin in patients with MBC and liver impairment.

\section{CONCLUSION}

Eribulin is a diverse and one-off anticancer agent, with both mitotic and nonmitotic MOAs, for the treatment of MBC after failure of therapy with an anthracycline and a taxane. Clinical trial data from phase III studies have demonstrated that eribulin has activity in difficult-to-treat patients of breast cancer, including those with pretreated HER2-negative disease or TNBC. Safety analyses were similar to other microtubule-targeting antimitotic agents, and additional safety data highlighted the potential of eribulin treatment in specific patient populations.

Funding: No funding sources Conflict of interest: None declared

Ethical approval: Not required

\section{REFERENCES}

1. Worldwide cancer research fund. Fact sheet: worldwide cancer data, 2015. Available at: http://www.wcrf.org/cancer_statistics/world_cancer _statistics.php. Accessed on 12 January 2021.

2. Mermer G, Turk M. Assessment of the effects of breast cancer training on women between the ages of
50 and 70 in Kemalpasa, Turkey. Asian Pac J Cancer Prev. 2014;15(24):10749-55.

3. Leśniczak B, Krasomski G, Oszukowski P, Stetkiewicz T, Woźniak P. Incidence of and mortality from breast cancer among women in Poland in the years 2001-2010. Prz Menopauzalny. 2014;13(6):344-7.

4. Ferrini K, Ghelfi F, Mannucci R, Titta L. Lifestyle, nutrition and breast cancer: facts and presumptions for consideration. Ecancermedicalscience. 2015;9:111.

5. Castelló A, Martín M, Ruiz A, Casas AM, BaenaCañada JM, Lope V, et al. Lower breast cancer risk among women following the world cancer research fund and american institute for cancer research lifestyle recommendations: epigeicam case-control study. PLoS One. 2015;10(5):1-15.

6. Dybdal-Hargreaves NF, Risinger AL, Mooberry SL. Eribulin mesylate: mechanism of action of a unique microtubule-targeting agent. Clinic Canc Res. 2015;21(11):2445-52.

7. Garrone O, Miraglio E, Vandone AM, Vanella P, Lingua D, Merlano MC. Eribulin in advanced breast cancer: safety, efficacy and new perspectives. Future Oncol. 2017;13(30):2759-69.

8. Jordan M, Kamath K, Manna T, Okouneva T, Miller HP, Davis C. The primary antimitotic mechanism of action of the synthetic halichondrin E7389 is suppression of microtubule growth. Mol Cancer Ther. 2005;4:1086-95.

9. Shetty N, Gupta S. Eribulin drug review. South Asian J Cancer 2014;3(1):57-9.

10. Agoulnik SI, Kawano S, Taylor N, Oestreicher J, Matsui J, Chow J, et al. Eribulin mesylate exerts specific gene expression changes in pericytes and shortens pericyte-driven capillary network in vitro. Vasc cell. 2014;6(1):1.

11. Funahashi Y, Okamoto K, Adachi Y, Semba T, Uesugi M, Ozawa Y, et al. Eribulin mesylate reduces tumor microenvironment abnormality by vascular remodeling in preclinical human breast cancer models. Cancer Sci. 2014;105(10):1334-42.

12. Yoshida T, Ozawa Y, Kimura T, Sato Y, Kuznetsov $\mathrm{G}, \mathrm{Xu} \mathrm{S}$, et al. Eribulin mesilate suppresses experimental metastasis of breast cancer cells by reversing phenotype from epithelial-mesenchymal transition (EMT) to mesenchymal-epithelial transition (MET) states. $\mathrm{Br} \mathrm{J}$ Cancer. 2014;110(6):1497-505.

13. O'Shaughnessy J, Kaklamani V, Kalinsky K. Perspectives on the mechanism of action and clinical application of eribulin for metastatic breast cancer. Future Oncol. 2019;15(14):1641-53.

14. Towle MJ, Salvato KA, Budrow J, Wels BF, Kuznetsov G, Aalfs KK, et al. In vitro and in vivo anticancer activities of synthetic macrocyclic ketone analogues of halichondrin B. Cancer Res. 2001;61(3):1013-21.

15. Morgan RJ, Synold TW, Longmate JA, Quinn DI, Gandara D, Lenz HJ, et al. Pharmacodynamics (PD) 
and pharmacokinetics (PK) of E7389 (eribulin, halichondrin $\mathrm{B}$ analog) during a phase $\mathrm{I}$ trial in patients with advanced solid tumors: a California cancer consortium trial. Cancer Chemother Pharmacol. 2015;76(5):897-907.

16. US FDA. Fact sheet: Highlights of prescribing information, 2010. Available at: https://www.accessdata.fda.gov/drugsatfda_docs/lab el/2010/201532lbl.pdf. Accessed on 12 January 2021.

17. Zhang ZY, King BM, Pelletier RD, Wong YN. Delineation of the interactions between the chemotherapeutic agent eribulin mesylate (E7389) and human CYP3A4. Cancer Chemother Pharmacol. 2008;62(4):707-16.

18. Devriese LA, Witteveen PO, Marchetti S, MerguiRoelvink M, Reyderman L, Wanders J, et al. Pharmacokinetics of eribulin mesylate in patients with solid tumors and hepatic impairment. Cancer Chemotherap Pharmacol. 2012;70(6):823-32.

19. Tan AR, Sarantopoulos J, Lee L, Reyderman L, He Y, Olivo M, et al. Pharmacokinetics of eribulin mesylate in cancer patients with normal and impaired renal function. Cancer Chemotherap Pharmacol. 2015;76(5):1051-61.

20. Kurebayashi J, Kanomata N, Yamashita T, Shimo T, Moriya T. Antitumor and anticancer stem cell activities of eribulin mesylate and antiestrogens in breast cancer cells. Breast Cancer. 2016;23(3):42536.

21. David L, Luu TH, Xing Q, Han ES, Yip R, Yim JH. Effect of eribulin on cell growth and PI3K pathway activity with and without RAD001 in triple-negative and HER2-expressing breast cancer. J Clinic Oncol. 2013;31(26): 173 .

22. Tan AR, Rubin EH, Walton DC, Shuster DE, Wong YN, Fang F, et al. Phase 1 study of eribulin mesylate administered once every 21 days in patients with advanced solid tumors. Clin Cancer Res. 2009;15(12):4213-9.

23. Towle MJ, Salvato KA, Budrow J, Wels BF, Kuznetsov G, Aalfs KK, et al. In vitro and in vivo anticancer activities of synthetic macrocyclic ketone analogues of halichondrin B. Cancer Res. 2001;61(3):1013-21.

24. Aoqi K, Iwata H, Masuda N, Mukai H, Yoshida M, Rai Y, et al. A phase 2 study of eribulin in Japanese patients with heavily pretreated metastatic breast cancer. Ann Oncol. 2012;23(6):1441-8.

25. Cortes J, Vahdat L, Blum JL, Twelves C, Campone M, Roché H, et al. Phase II Study of the Halichondrin $\mathrm{B}$ analog eribulin mesylate in patients with locally advanced or metastatic breast cancer previously treated with an anthracycline, a taxane, and capecitabine. J Clin Oncol. 2010;28(25):3922-8.

26. Vahdat LT, Pruitt B, Fabian CJ, Rivera RR, Smith DA, Tan-Chiu E, et al. Phase II study of eribulin mesylate, A halichondrin B analog, in patients with metastatic breast cancer previously treated with an anthracycline and a taxane. J Clin Oncol. 2009;27(18):2954-61.

27. Cortes J, O'Shaughnessy J, Loesch D, Blum JL, Vahdat LT, Petrakova K, et al. Eribulin monotherapy versus treatment of physician's choice in patients with metastatic breast cancer (EMBRACE): a phase 3 open-label randomised study. Lancet. 2011;377(9769):914-23.

28. Muss H, Cortes J, Vahdat LT, Cardoso F, Twelves C, Yang $\mathrm{J}$, et al. Eribulin monotherapy in patients aged 70 years and older with metastatic breast cancer. Oncologist. 2014;19(4):318.

29. Kaufman PA, Awada A, Twelves C, Yelle L, Perez EA, Velikova G, et al. Phase III open-label randomized study of eribulin mesylate versus capecitabine in patients with locally advanced or metastatic breast cancer previously treated with an anthracycline and a taxane. J Clin Oncol. 2015;33(6):594.

30. Twelves C, Cortes J, Vahdat L, Olivo M, He Y, Kaufman PA, et al. Efficacy of eribulin in women with metastatic breast cancer: a pooled analysis of two phase 3 studies. Breast Cancer Res Treat. 2014;148(3):553-61.

31. Pivot X, Marmé F, Koenigsberg R, Guo M, Berrak E, Wolfer A. Pooled analyses of eribulin in metastatic breast cancer patients with at least one prior chemotherapy. Ann Oncol. 2016;27(8):1525-31.

32. Barni S, Livraghi L, Morritti M, Vici P, Michelotti A, Cinieri S, et al. Eribulin in the treatment of advanced breast cancer: real-world scenario from 39 Italian centers-ESEMPiO study. Future Oncol. 2019;15(1):33-44.

33. Fabi A, Moscetti L, Ciccarese M, Caramanti M, Salesi N, Verde NL, et al. Eribulin in heavily pretreated metastatic breast cancer patients and clinical/biological feature correlations: impact on the practice. Future oncol. 2015;11(3):431-8.

34. Leo S, Arnoldi E, Repetto L, Coccorullo Z, Cinieri S, Fedele $\mathrm{P}$, et al. Eribulin mesylate as third or subsequent line chemotherapy for elderly patients with locally recurrent or metastatic breast cancer: a multicentric observational study of GIOGer (Italian group of geriatric oncology)-ERIBE. Oncologist. 2019;24(6):232.

35. Dranitsaris G, Gluck S, Faria C, Cox D, Rugo H. Comparative effectiveness analysis of monotherapy with cytotoxic agents in triple-negative metastatic breast cancer in a community setting. Clinical Therapeut. 2015;37(1):134-44.

36. Kish JK, Mougalian SS, Copher R, McAllister L, Zhixiao W, Broscious M. Abstract P5-15-16: Utilization and outcomes of eribulin in triple negative metastatic breast cancer: Real-world findings. Cancer Res. 2017:5-15.

37. Watanabe J. Eribulin monotherapy improved survivals in patients with ER-positive HER2negative metastatic breast cancer in the real world: a single institutional review. Springerplus. 2015;4(1):1-6. 
38. Wilks S, McIntyre K. Case studies in the management of metastatic breast cancer with eribulin. Clinic Med Insight: Oncol. 2015;9:27962.

39. Lorusso V, Cinieri S, Latorre A, Porcu L, Del-Mastro L, Puglisi F, et al. Efficacy and safety of eribulin in taxane-refractory patients in the 'real world'. Future Oncol. 2017;13(11):971-8.

40. Matsuoka H, Tsurutani J, Tanizaki J, Iwasa T, Komoike Y, Koyama A, et al. Regression of brain metastases from breast cancer with eribulin: a case report. BMC Res. 2013;6(1):1-4.

41. Chang AY, Ying XX. Brain metastases from breast cancer and response to treatment with eribulin: a case series. Breast Cancer: Basic Clinic Res. 2015;9:21176.

42. Kish JK, Mougalian SS, Copher R, McAllister L, Wang Z, Janevski M, et al. Utilisation and outcomes of eribulin in triple-negative metastatic breast cancer (tn mbc): real-world findings. Asia Pac J Clinic Oncol. 2017;13:123-123.

43. Byun KD, Ahn SG, Baik HJ, Lee A, Bae KB, An MS, et al. Eribulin mesylate combined with local treatment for brain metastasis from breast cancer: two case reports. J Breast Canc. 2016;19(2):214.

44. Yoshinami T, Masuda N, Nakayama T, Hara F, Sagara Y, Kawaguchi H, et al. The utility of biweekly eribulin therapy for metastatic breast cancer: a Japanese multicenter Phase II study (JUSTSTUDY). J. Clin. Oncol. 2015;33:1026.

45. Ohtani S, Nakayama T, Yoshinami T, Watanabe KI, Hara F, Sagara Y, et al. Bi-weekly eribulin therapy for metastatic breast cancer: a multicenter phase II prospective study (just-study). Breast Cancer. 2018;25(4):438-46.

46. Vahdat LT, Garcia AA, Vogel C, Pellegrino C, Lindquist DL, Iannotti N, et al. Eribulin mesylate versus ixabepilone in patients with metastatic breast cancer: a randomized Phase II study comparing the incidence of peripheral neuropathy. Breast Canc Res Treat. 2013;140(2):341-51.

47. Goel S, Mita AC, Mita M, Rowinsky EK, Chu QS, Wong N, et al. A phase I study of eribulin mesylate (E7389), a mechanistically novel inhibitor of microtubule dynamics, in patients with advanced solid malignancies. Clinic Cancer Res. 2009;15(12):4207-12.

Cite this article as: Gadekar A. Eribulin: clinical review and new perspective in advanced breast cancer. Int J Adv Med 2021;8:721-7. 